Journal of Advanced Research in Fluid Mechanics and Thermal Sciences

Journal homepage: www.akademiabaru.com/arfmts.html ISSN: 2289-7879

\title{
Aerodynamic Architectural Features to Mitigate Peak Pressures over Low- Rise Buildings
}

\author{
Yasser M. El-Okda ${ }^{1,}{ }^{*}$, Mohamed S. Emeara² \\ Mechanical Engineering Department, Higher Colleges of Technology, Abu Dhabi Men's College, Abu Dhabi, United Arab Emirates \\ Department of Mechanical Power Engineering, Zagazig University, Zagazig 44519, Egypt
}

\begin{tabular}{ll} 
ARTICLE INFO & ABSTRACT \\
\hline $\begin{array}{l}\text { Article history: } \\
\text { Received } 5 \text { May } 2021\end{array}$ & $\begin{array}{l}\text { A number of aerodynamic devices to perturb or modify the flow over low-rise buildings } \\
\text { are proposed. The purpose of these devices is to reduce the adverse effects of wind } \\
\text { Received in revised form } 10 \text { June } 2021\end{array}$ \\
$\begin{array}{l}\text { Accepted } 15 \text { June } 2021 \\
\text { Available online } 8 \text { July } 2021\end{array}$ & $\begin{array}{l}\text { levels. Pressure measurements were performed on a scaled down model of the well- } \\
\text { known TTU experimental building of the Wind Engineering Research Facility (WERF) } \\
\text { Keywords: }\end{array}$ \\
$\begin{array}{l}\text { at TTU University. Different results were obtained: reduction of average peek vacuum, } \\
\text { smoothing of windward pressure distribution on the roof and narrowing and localizing } \\
\text { prism; building aerodynamics; building } \\
\text { architectural features }\end{array}$ & $\begin{array}{l}\text { disciplines such as heat transfer are also reported. } \\
\text { lofing }\end{array}$
\end{tabular}

\section{Introduction}

A major issue for low-rise buildings is wind loading. Winds not only impose structural loading on buildings, yet they also erode the building surficial elements such as facades and roofs. The outside building walls undergo extreme wind fluctuations making it highly vulnerable to failure by fatigue. This effect is more pronounced on the roof of the building where a fluctuating negative pressure field exists. This fluctuating negative pressure field weakens building roofs and leads to highly expensive damage to low-rise building as well as dangers.

Extensive research has been done to study the flow over low-rise buildings. Nevertheless, it can be said that the majority of the work done contributed to the understanding of the problem and the flow regime. Most of the work focused mainly on characterizing the problem, correlating flow field variables, aerodynamic testing of urban building complexes, statistical analysis of temporal pressure measurements, assessing or modelling of the wind load on building structures, validation of numerical codes, and testing of accuracy of turbulence models in predicting the complex flow field. There aren't enough studies that included modification or addition of architectural features to improve building aerodynamic performance.

\footnotetext{
* Corresponding author.

E-mail address: yelokda@hct.ac.ae

https://doi.org/10.37934/arfmts.84.2.2438
} 
Many of the studies done to investigate the wind effect over low-rise buildings adopted a plain prism, cube mounted to a ground floor or similar geometries as a working model with different dimensions and orientations with respect to the mean stream [1,2]. Roughness elements or rough wall models are usually employed upstream before the building to simulate rough terrain as conducted in a study by Aboshosha et al., [3]. Unsteady computations are conducted by Blocken et al., [4] on cubic model. The work of Yu et al., [5] studied the influence of aspect ratio on the flow around a prism using LES. In a study by Kwon and Kareem [6], they introduced the "Gust Front Factor" to account for transient aerodynamic effects in building codes. The effect of turbulence on the flow over surface-mounted prism using LES accompanied by velocity field measurements via PIV is examined by El-Okda et al., [7]. An experimental investigation was done by Lim et al., [8] where pressure measurements and smoke flow visualizations were done on a cube.

The flow over low-rise building or surface mounted prism is one of the well investigated problems although not yet quite understood. A vast literature can be found on this problem. Therefore, we shall focus on architectural features introduced to low-rise buildings or likewise structures.

The present research was carried out in the Mechanical Power Engineering Laboratory at the Faculty of Engineering, Zagazig University, during the summer vacation for the year 2018.

\section{Architectural Features}

Many studies can be found in literature where architectural features were introduced to a structure placed on ground and subject to cross wind. The work of Liu et al., [9] studied the pressure characteristics around a pyramid located behind a porous fence. They found that by installing the porous fence, the mean pressure and pressure fluctuations on the pyramid were decreased. In addition, the porous fence dissipates part of the kinetic energy of the flow as well as breaks turbulent structures down close to dissipative scales. Hence, the effect of large coherent structures, developing upon flow separation on the structure façade, is reduced. They also found that installing another fence behind the pyramid reduces the pressure fluctuations into half. In a study by Baskaran et al., [10], they reported that the effect of parapets, commonly employed in buildings, is among the factors that are critical to the failure of the roofing systems. Research work by llgın and Günel [11] presented a good review on building aerodynamic modification applied to tall buildings. They classified such modifications as major or minor according to their effect on the architectural design. The approach of Kumar [12] adopted an inclined gable roof steeping building as their model in studying wind load on buildings with overhang, building aspect ratio, and building with boundary wall all around. They also studied effect of terrain topology, mainly building over hill. The experimental study by John et al., [13] investigated wind load on the roof of a gable building with an overhang and with a boundary wall. They found that when using a boundary wall at two to four times the building height, suction on the roof is reduced. The experimental work of Soleimani et al., [14] conducted a study of the air flow over a doomed roof. An interesting and comprehensive structural and architectural perspective on low-rise buildings in wind is exhibited by Zhao and He [15]. Among these building configurations are: flat roof, pitched roof, roofs with eaves and overhangs. The author suggested that an opening on the roof helps reduce the uplift. Additionally, roofs with multiple slopes, e.g., hip roof, show better aerodynamic performance than gable roof (two-slope roof). It is also stated that for roofs with long overhang, it is recommended to have an opening in the roof at the junction of the wall with the overhang. Research work of Shamansurov and Sharipov [16] proposed and tested several successful modifications to truck-trailer assembly. Their work resulted in reduction of the aerodynamic drag on the assembly that resulted in significant fuel economy. It should be noted that there are some major differences between the flow over low-rise building and the flow over trucks. These differences 
appear mainly in: inflow conditions (boundary layer) due to vehicle motion, the underbody gap, the gap between the truck and the trailer, the moving ground, and the rolling wheels.

\section{Building Roof Geometries}

\subsection{Functional Features}

Along with structural and architectural building roof modifications, there exist modifications of functional purpose. The work of Meroney and Neff [17] studied the flow over a row of solar collectors in saw teeth arrangement placed on the top of a building model. A turbine is installed at the top of the roof where the two slopes meet as shown in Figure 1. The design is such that part of the airflow over the roof flows through the gap between the two layers providing the necessary momentum to run the turbine. The rest of the air passing around the roof helps posing a driving potential for the air flow exiting the turbine by the accelerating airflow on the roof. Hence increasing the velocity and reducing the pressure around the turbine exit. Although it is a decent utilization of a building roof, its use for producing electrical energy is limited as the incoming flow momentum at such heights within the atmospheric boundary layer is relatively low.
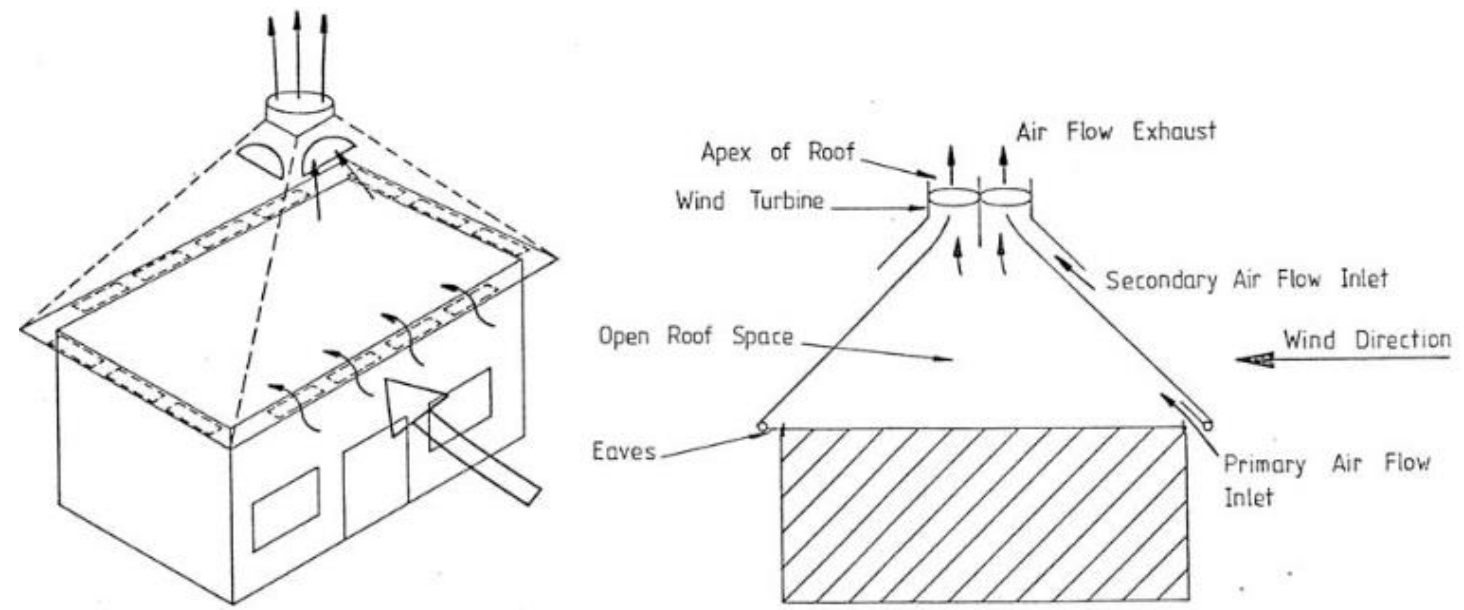

Fig. 1. Roberstson and Leaman's Roof Turbine Concept [18]

\subsection{Flow Field over Low-Rise Building}

In this section, the main features of the flow over a low-rise building are described for a plain prism building model. The incident flow initially undergoes secondary separation on the ground ahead of the building. This secondary separation is of minor effect on the flow field. The flow then continues to hit the building façade. Stagnation occurs over a line on the façade that extends horizontally over the full span of the building as shown by El-Okda [19]. Part of the flow assumes a downward direction below the stagnation line and rolls ahead of the building resulting in a large vortex that extends around the building defining what is known as the horseshoe vortex. This part of the flow eventually passes around the building side walls. The rest of the flow moves upward above the stagnation line. The upward flow accelerates on the façade at a relatively high-speed making a wall jet. Shorty, this flow separates after at the windward edge of the building and assumes a nonlinear inclined path with the mean flow above the roof. This nonlinearity is attributed to the mono sign vorticity in the separating shear layer as well as continual forcing of the shear layer by the incident flow as we move farther upward away from the roof. Span-wise vortices develop on the front façade of the building [19]. They advect with the separating flow above the building roof. Such 
shear flow involves set off by the jet-like flow of the shear layer perturbation by the mono sign vorticity. By the effect of the shear layer instability and the effect of the vorticity field, some of the shedding vortices periodically depart from the shear layer towards the roof. It should be noted that the departing vortices leave the shear layer strictly towards the roof. This is because of the following two reasons. First, the velocity gradient from below the shear layer is larger than the velocity gradient from above the shear layer. This is because of the existence of the roof surface. Second, the incident flow approaching the shear layer from above further decreases the velocity gradient and hence stabilizes the shear layer from this side. It worth noting here that the momentum of incident flow from above the shear layer damps down perturbations in this side of the layer hindering any vortices moving with shear layer from escaping from the upper side of the shear layer.

The vortices leaving the shear layer and hitting the roof have relatively low pressure in their cores. The peak vacuum pressure measured on the roof is due to the proximity of these vortices to the surface. A thin vortex, evolving with the separating flow, would probably possess high vorticity and relatively low pressure in the core. In this case, higher vacuum pressure may be observed on the roof than that of a larger size vortex.

In a study by Josserand and Rossi [20], a description of the merging mechanism of two co-rotating vortices where the two vortices roll together around each other until the merging is completed. In our case, the shed vortices are affected by other periodically shed vortices as well as by the proximity to the roof surface. The separating shear flow is unstable such that a newly shed vortex may drift towards the roof and keeps rolling towards the roof by the effect of the strong vorticity in the shear layer. A co-moving vortex could have merged with it if the roof is not present. As a vortex rolls towards the roof, it clears the way to its subsequent one to be convected with the shear layer. Hence, the latter vortex facing the roof is hindered from continuing any possible merging with its preceding or subsequent (or following) vortices.

The flow field around a bluff body, in our case the surface mounted prism, is highly complex and three-dimensional. Of high significance flow patterns are: the incident boundary layer (affects stagnation level on the building façade and thus the flow separation at the roof), streamline curvatures (creating instabilities that generates streamwise vortices), and a shear layer (convects continuously shed vortices upon flow separation). The shed vortices after separation, interact with each other and with the roof surface. The mean flow field does hide such unsteady interactions. "Steady computations produce an erroneously long wake because they omit the periodic vortex shedding" [21]. To adopt any effective architectural modifications, the real flow field is the one that should be considered before any new configuration is proposed. The separation bubble on the roof is such a good example to unsteady interactions concealed within the time averaged flow field. This separation bubble is averaged from much smaller scale eddies that either convect with the shear layer, setting the upper boundary of the separation bubble, or revert, after hitting the roof, setting the lower boundary of the separation bubble. Hence, the mean separation bubble hides all of these interactions making it less constructive to depend on them to build any new significant modification. In addition to considering the aforementioned flow patterns and the unsteady flow field, it is also more effective to consider instabilities that may commence and develop with such complex flows.

According to the forgoing description of the flow at around a low-rise building, the author is adopting the following bases for his proposed architectural modifications

(i) Vortex stretching and contraction via axial straining and vortex tilting and stretching via lateral straining. Hence, accelerating the flow above the roof while forcing the flow to pass through a converging arrangement. It is expected that roof vortices are forced to contract, thus increasing their sizes, which elevates the vortex core away from the roof surface. In the meantime, the flow assumes upward velocity as a result of flow blockage. Additionally, as the 
flow accelerates with the converging passage, it undergoes lateral straining accompanied by streamwise stretching of the vortex filaments. Such straining disrupts the typical vortical interactions on the roof that brings vortices close to the roof. Highly strained structures eventually break down into parcels of small-scale vorticity. The result is reduction in the vacuum pressure on the roof and more uniformity of the pressure distribution as shown later.

(ii) Breaking of vortices into smaller eddies. These strong coherent structures on the roof are cut by thin plates or blades oriented parallel to the freestream. These structures, after being cut are then subject to a rapid straining due to the sudden introduction of high shearing rate. Further straining of these vortices may lead to their breakdown.

(iii) Hindering the vortices from reaching the roof by: employing a wing-shaped device at low angle of attack ahead of the building, and forcing more of the mainstream flow to roll downward and finally get entrained with the horseshoe vortex. The wing-shape device has a vorticity field and poses downwash on the wake behind the trailing edge, therefore, giving the flow leaving the wing a downward velocity component. Streamwise vortices in the wake would favor an inclined path due to the inherent instability accompanying streamline curvature. All of these factors pour into an increased mass flow around the building from around the sides.

(iv) Forcing more of the incident flow to assume a path around the building instead of flowing over the roof. It is shown by El-Okda [19] that the flow hitting the building façade, on average, which passes below the stagnation line, is essentially composed of the mass flow in the boundary layer of the incident flow. This means that most on the incoming flow hits the façade and generate a high momentum boundary layer above the stagnation line that separates at the edge of the façade. Hence, the idea is to force part of the main flow ahead of the façade to follow a downward path. This elevates the stagnation point, reduces the upward flow on the façade. The effect of that is reduction in the momentum of the separating flow and directing the turning-down flow to roll around the building with the horseshoe vortex.

\section{Experimental Setup}

A schematic of the wind tunnel used in the experiments is shown in Figure 2. The test section is of $0.5 \mathrm{~m} \times 0.5 \mathrm{~m}$ cross section and is $2 \mathrm{~m}$ long. The model is placed at $0.6 \mathrm{~m}$ before the tunnel exit. A Preston static tube was used at the middle of the test section to measure the freestream velocity without the model in place. No roughness elements were used on the tunnel floor upstream of the model. The turbulence intensity is about $7 \%$. The boundary layer profile, Figure 3 , was obtained using Preston tube.

The model is a scaled down replica of the well-known Texas Tech University (TTU) building. The model height $(H)$ : width $(W)$ : length $(L)$ is 1:2.725:3.425 with height of 0.038 m. Figure 3 shows an unfolded image of the model. The grid nodes in the figure represent the locations of the pressure taps used for measurements. The freestream direction is from left to right in all of the following plots of the pressure distribution. The incident flow velocity at the model height is $13.8 \mathrm{~m} / \mathrm{s}$ as marked by a dash line in Figure 4. The Reynolds number based on the model height is 35000. 


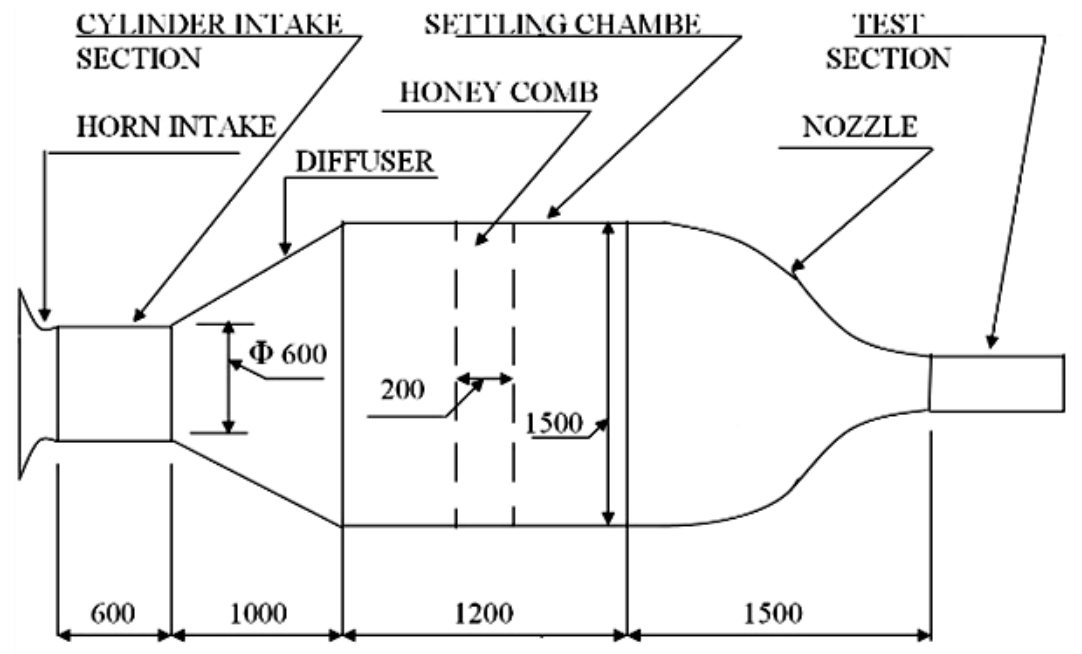

Fig. 2. A schematic of the wind tunnel (dimensions are in $\mathrm{mm}$ )

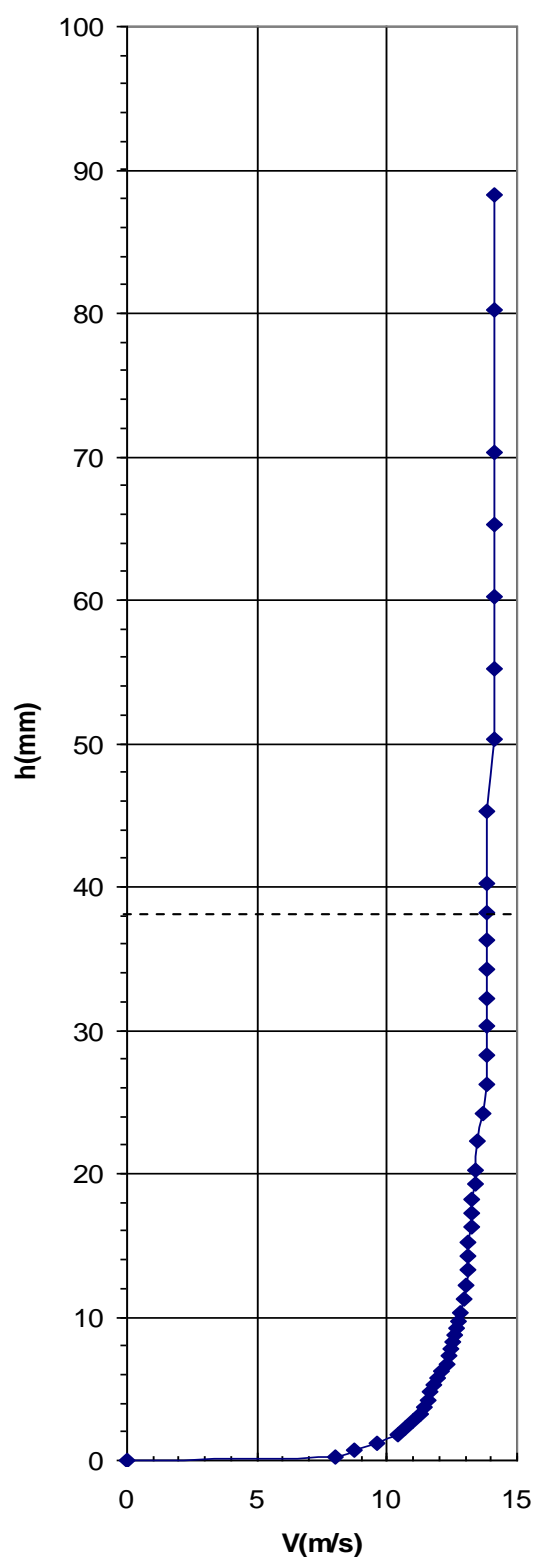

Fig. 3. Boundary layer profile 


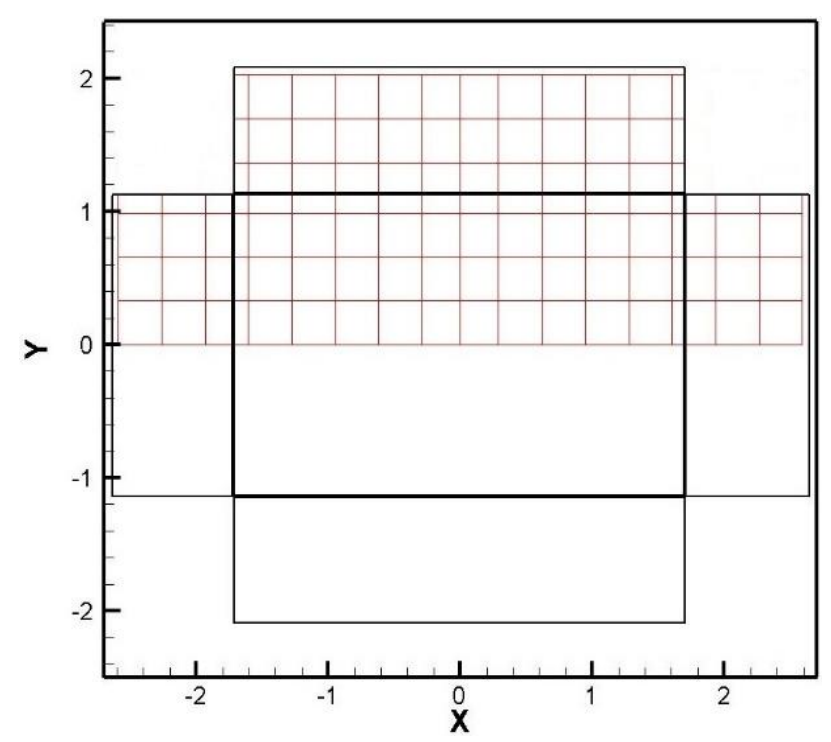

Fig. 4. Pressure tap grid on an unfolded model

\subsection{Experimental Verification}

Pilot pressure measurements were taken on a bare model without any modifications. The pressure distribution was compared to published results by El-Okda et al., [7]. The resulting pressure distribution, Figure 5, did not match any published results. The same experiment was repeated while with the free ends of all tubing used in the measurement were closed. The resulting pressure distribution matches well with published work as shown in Figure 6 . A delivery tunnel was used during the experiments. This poses excess pressure above ambient throughout the tunnel. Having 82 pressure taps on the model surface, makes it vulnerable to significant flow through the taps as measurements are taken one tap at a time. This situation highly spoils the flow over the model surface which affects pressure distribution.

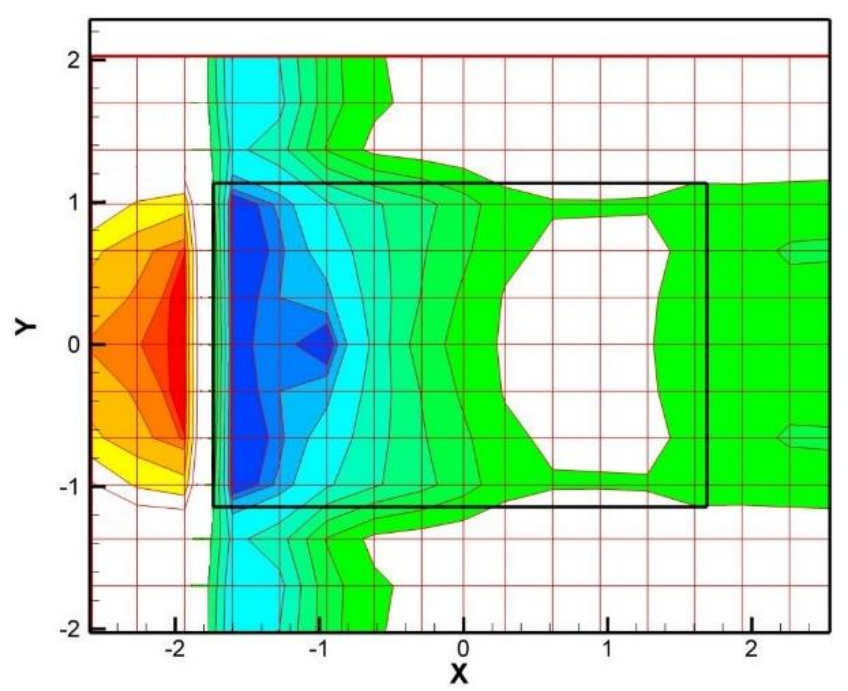

Fig. 5. CP on the surface of the model with open ended pressure taps, flow from left to right 


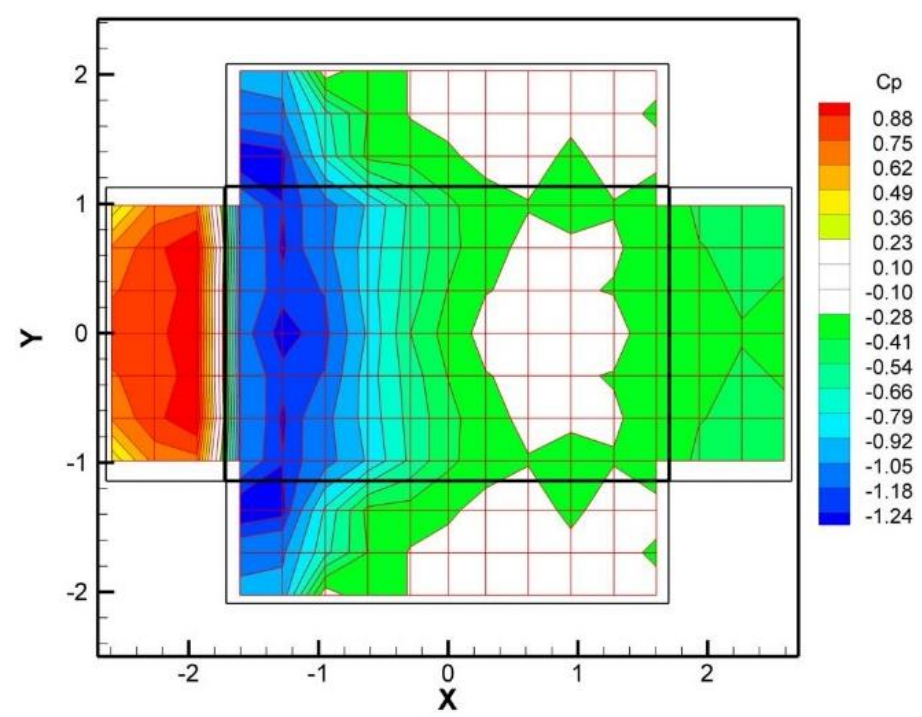

Fig. 6. CP on the surface of the model with closed idle pressure taps, flow is from left to right

\section{Results and Discussion}

According to our understanding to the flow field over low-rise buildings, a number of models were created each is equipped with an architectural feature. Each of these features is expected to improve the pressure distribution on the roof. The pressure distribution could be adjusted in one of the following ways: reduction of the peak pressure on the roof, flattening of the pressure distribution, combination of aforementioned effects, and finally confining of the peak pressures to localized spots. We discuss each of these cases in the following.

A case where the building was equipped with a spoiler installed on the roof is presented in Figure 7. The spoiler frame blades make $30^{\circ}$ angle with the roof surface. It was expected that the spoiler frame would deviate the inflow away from the roof resulting in more uniform pressure distribution of low peak vacuum. Apparently, a strip of relatively high vacuum was developed behind the leading edge on the roof.

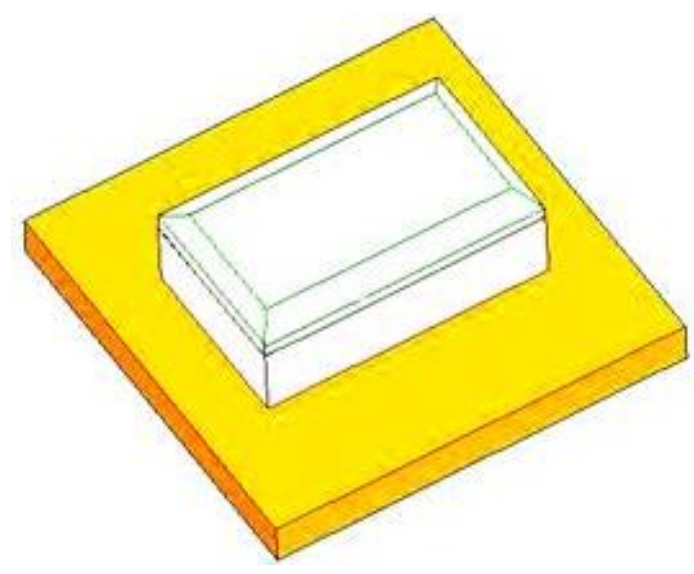

(a)

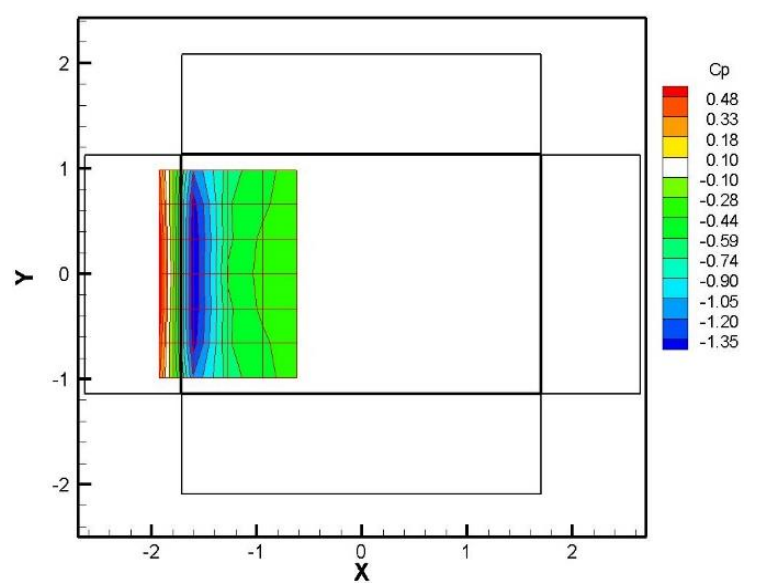

(b)

Fig. 7. Roof with an elevated spoiler frame making $30^{\circ}$ angle with the roof surface 
Figure 8(a) and Figure 9(a) show the building roof with two vertical plates installed on the roof at $30^{\circ}$ and $45^{\circ}$ to the side walls respectively. These plates are expected to alter the flow field as follows. First, they slightly block the flow so as to diverge the incident separating flow away from the roof. Second, the two plates converge the flow which may result in contraction of the separated span-wise vortices moving close to the roof. As a result, the vortices are expected to increase in size leading to possible elevation of the vortex cores and increase in the core pressure. The probability of high vacuum on the roof decreases. Third, the vortical structures convecting with the separated flow are expected to undergo high stretching by the streamwise flow enhanced by their reorientation of the in the streamwise direction. This is a result of the lateral strain imposed by the resulting spanwise velocity gradient. The end result is distortion of the organized flow in the separation shear layer. The flow-surface interactions are thus broken down leading to significant change in the velocity distribution on the roof. It worth noting that the vortices developing and convecting with the separating flow encounter strong straining and undergoes significant streamwise as well as lateral stretching. It can be seen from Figure $8(b)$ and Figure $9(b)$ that the peak vacuum pressures are swept to very narrow spots behind the separation edge on the roof. As the angle of the vertical plates with the side walls increase, less vacuum is observed and the peak vacuum assumes narrower areas on the roof.

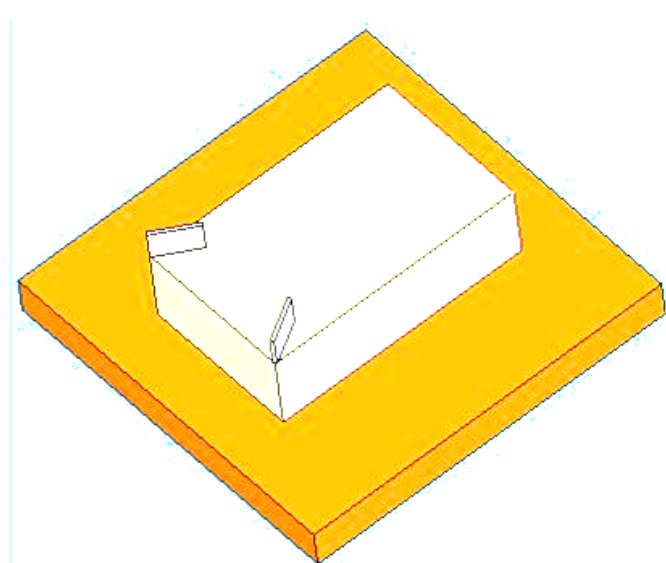

(a)

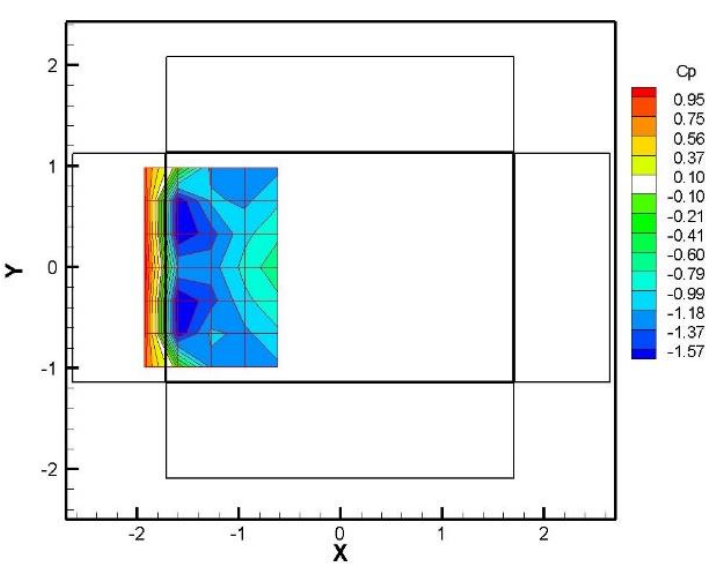

(b)

Fig. 8. Roof with two vertical rectangular plates, beginning at the front corners on the roof and at $30^{\circ}$ with the side walls

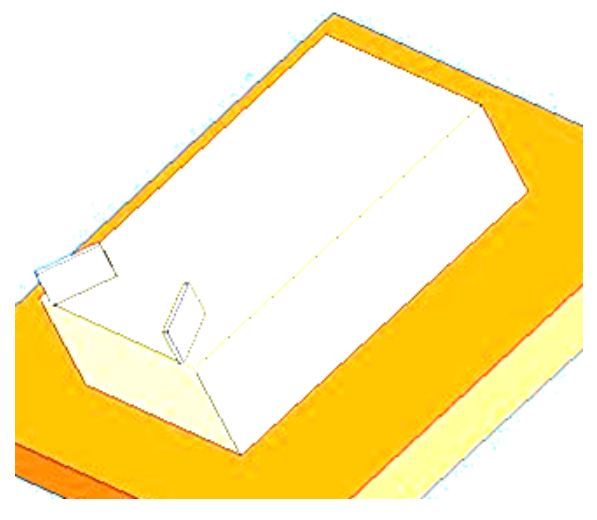

(a)

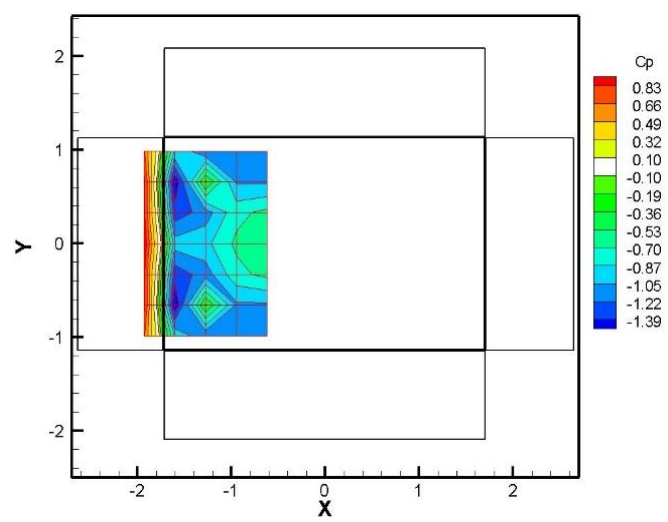

(b)

Fig. 9. Roof with two vertical rectangular plates, beginning at the front corners on the roof, and making $45^{\circ}$ to the side walls in the forward direction 
In Figure 10 and Figure 11, two pairs of vertical plates were installed symmetrically on the roof. The plates make $30^{\circ}$ and $45^{\circ}$ with the freestream as shown in Figure 10(a) and Figure 11(a) respectively. It should be noted that for the former case, the plates in each pair are separated by a distance while they meet in the latter case. Here we can see more profound effect of the blockage of these devices to the separating flow. The resulting pressure distributions for both cases are presented in Figure 10(b) and Figure 11(b). It is apparent that vacuum peaks are eliminated in the second case except for the narrow gap between the two pairs where the flow possesses high velocity that results in an intense low pressure stain around the line of symmetry behind the leading edge on the roof.

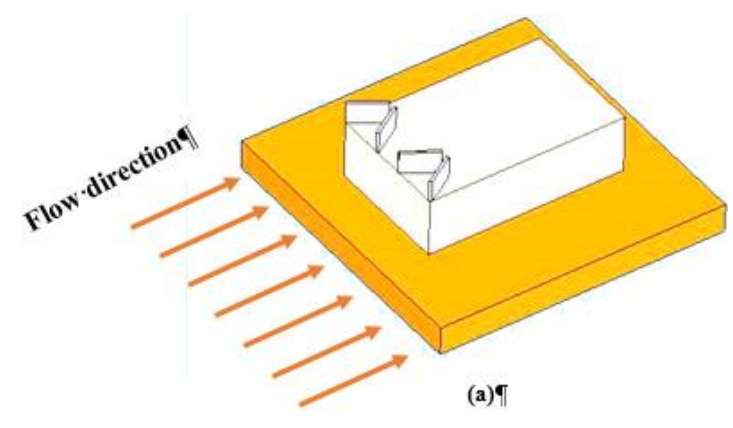

(a)

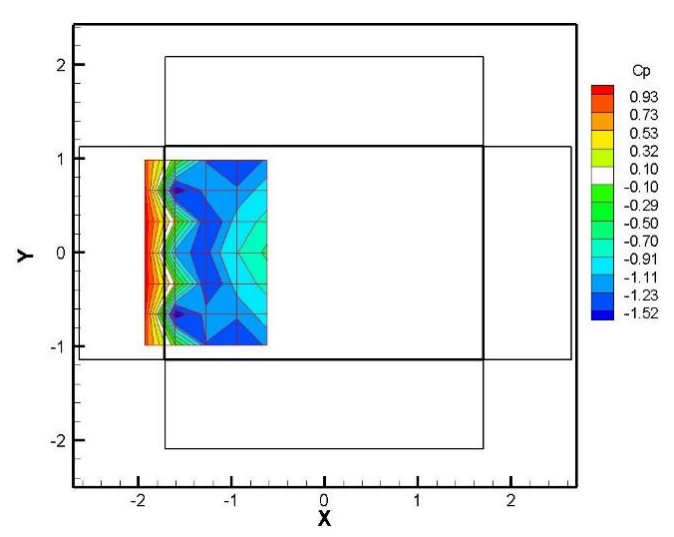

(b)

Fig. 10. Roof with two pairs of vertical rectangular plates behind the roof leading edge and each making $30^{\circ}$ with the incident flow

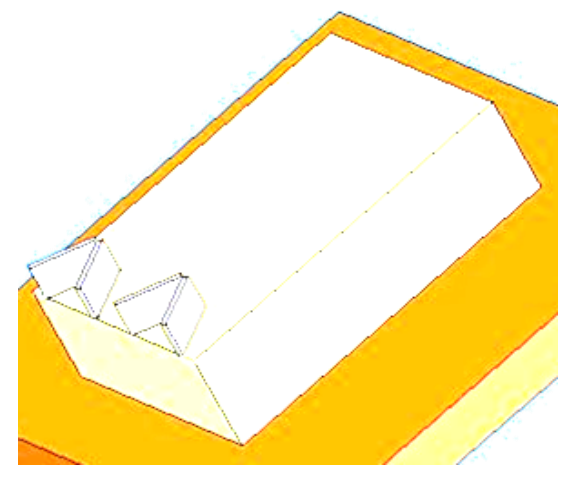

(a)

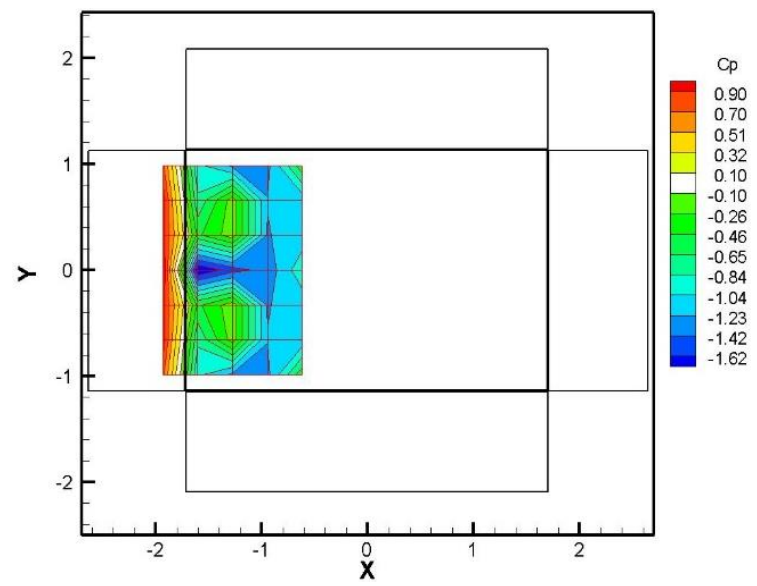

(b)

Fig. 11. Roof with two pairs of vertical rectangular plates behind the roof leading edge and each making $45^{\circ}$ with the incident flow

In the next two cases, shown in Figure 12 and Figure 13, an array of 6 blades is installed on the roof parallel to the mean flow. Two types of blades were employed, one in each case: triangular blades and rectangular blades. The separating flow is of dominant two-dimensional profile with spanwise vortices of relatively strong vorticity. After these vortices separate with the shear layer, they get cut by the blades. Due to the already existing strong vorticity within such structures, they may not develop streamwise vortices as it may occur in the usual channel flow. As the vortices are cut by the blades, they encounter high velocity gradient that makes them undergo very rapid and high stretching in a thin layer near the blades. Additionally, these vortices may experience twisting upon 
the sudden introduction of such high velocity gradient. Rapidly developing twisting poses an unstable state which may eventually leads to vortex breakdown.

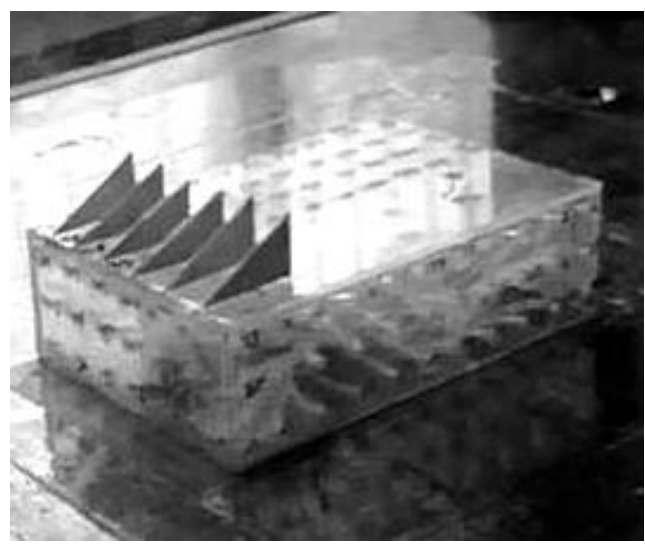

(a)

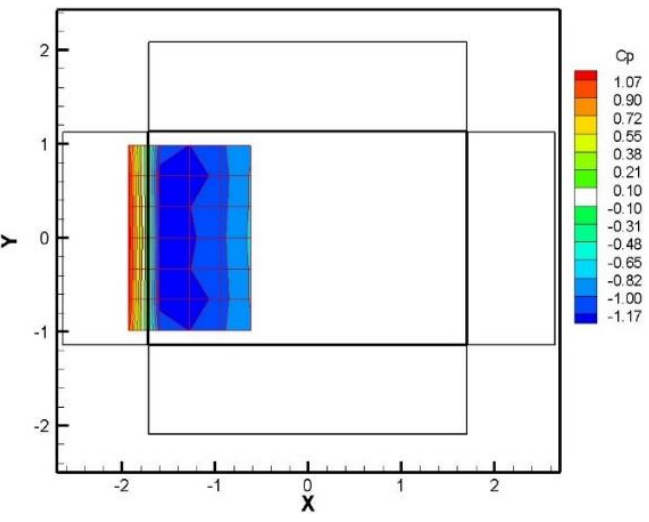

(b)

Fig. 12. roof with six $30^{\circ}$ right triangular blades streamlined with the mean flow and distributed on the roof behind the windward edge with (no blades at the corners)

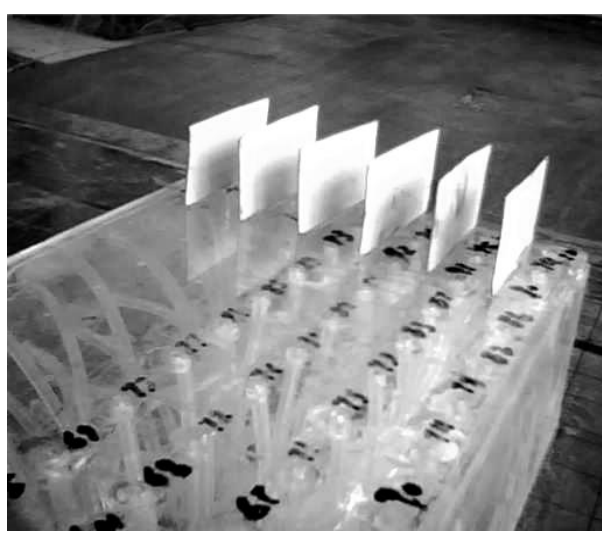

(a)

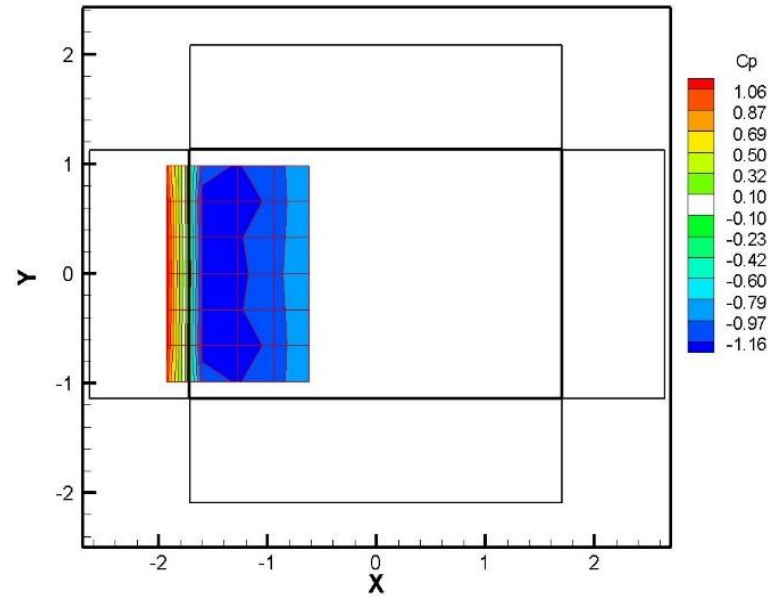

(b)

Fig. 13. Roof with six rectangular blades streamlined with the mean flow and distributed on the roof behind the windward edge with (no blades at the corners)

Figure 14 shows the results obtained after installing narrow curved strips to the leading edge of the triangular blades illustrated in Figure 12 . The $30^{\circ}$ inclination of these strips makes them roughly parallel to the mean direction of the separating flow. This provides for a filter for the convecting vortices from reaching the building roof as the strips block these vortices from passing to the roof. The strip curvatures allow the vortices to stretch rather than getting sheared upon approaching the strips. Additionally, these strips obstruct any vortical interactions taking place over a bare roof where of the vortices move backward upstream close to the roof surface after hitting the roof. It should be noted that the strip array poses a means for positive pressure gradient that helps reduce instabilities within such complex unsteady flow. The forgoing scenario needs more investigation. It is still possible that much of the flow is accelerated as it moves through the gaps between the strips. However, this may not be the case as the peak pressure on the roof is relatively low and uniformly distributed over a large area as shown in Figure 14 (b). It worth noting that the $\mathrm{Cp}$ at $0.8 \mathrm{H}$ on the façade has dropped to about 0.87 while it is about 1.06 in the preceding two cases. 


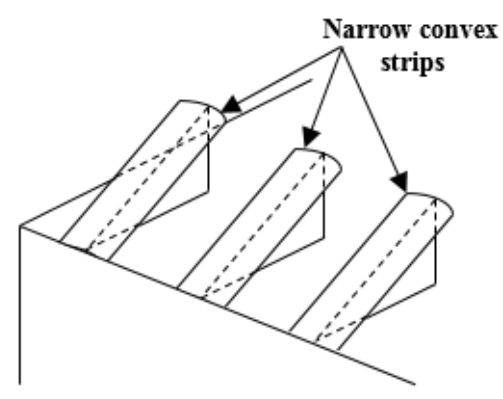

(a)

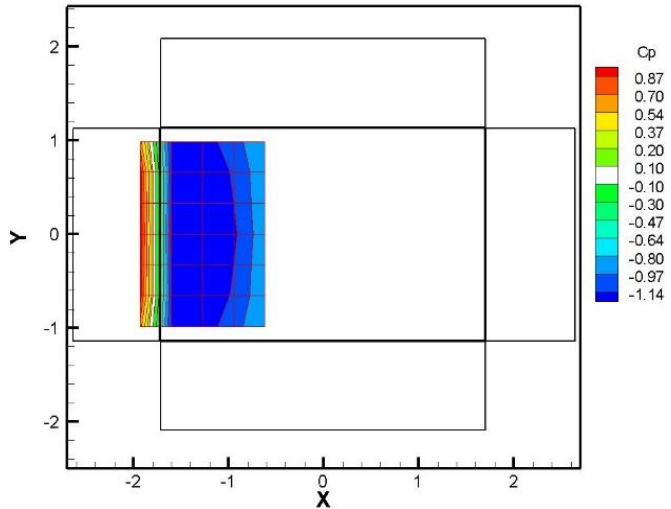

(b)

Fig. 14. Roof with six $30^{\circ}$ right triangular blades streamlined with the mean flow and distributed along the roof front edge. Each blade has a curved strip attached to its windward edge

Another experiment was conducted with rectangular blades installed to the upper part of the model façade and normal to the façade as shown Figure 15(a). It can be seen that a large area of uniform high vacuum pressure developed on the roof behind the building windward edge, Figure 15(b). As illustrated earlier, the flow reaches stagnation along a horizontal line on the façade. Part of the flow assumes upward direction above the stagnation line and the rest of the flow passes down joining the horseshoe vortex. In the current case, the upward flow on the façade is forced to pass through the narrow gaps bounded by the rectangular blades from the sides and by the façade from one other side of the passage. The resulting shear flow in the gap is thus of highly threedimensional perspective within the channel-like flow. The development of this flow field may be described as follows. The incident flow reaches stagnation on the building façade. A wall jet develops as the flow moves upward away from the stagnation line. As the flow passes in the passage between two plates, a pair of streamwise counter rotating vortices develops due to inflectional instability backed by Görtler instability resulting from the high curvature of the turning flow, The resulting longitudinal vortices in the boundary layer are amplified by the curvature and the rate of change of $|r V|$ with $r$ is decreasing in the vicinity of the stagnation region. Where $r$ and $V$ are the radius of curvature and flow velocity along the curvature respectively. These instabilities overpower the Kelvin-Helmholtz instability that may commence in the free shear flow of mostly two-dimensional perspective as in the flow over the bare façade. These streamwise vortices are shielded by being lifted away from the façade by means of the inward motion between the two counter-rotating vortices. In addition, these streamwise vortices are of relatively small scale affected by the gap between the plates, they keep stretching very close to the roof surface after separation. High vacuum is thus expected on the roof as a result of these thin high vorticity vortices that spread at a high proximity to the roof surface. It can be seen in Figure 15(b) that there is a spot of relatively higher pressure at the front corners on the roof. These are the spots corresponding to the outmost separated flow on the façade where the flow is not confined between vertical blades.

Although this configuration is of less interest to low-rise buildings, it may be of particular interest to vehicle aerodynamics and of more importance towards enhancing heat transfer providing fins as well as positive flow modifications. 


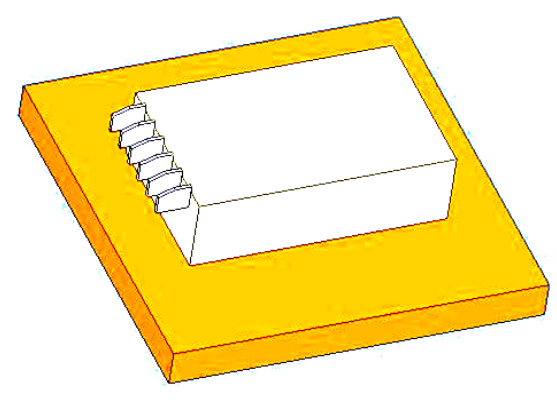

(a)

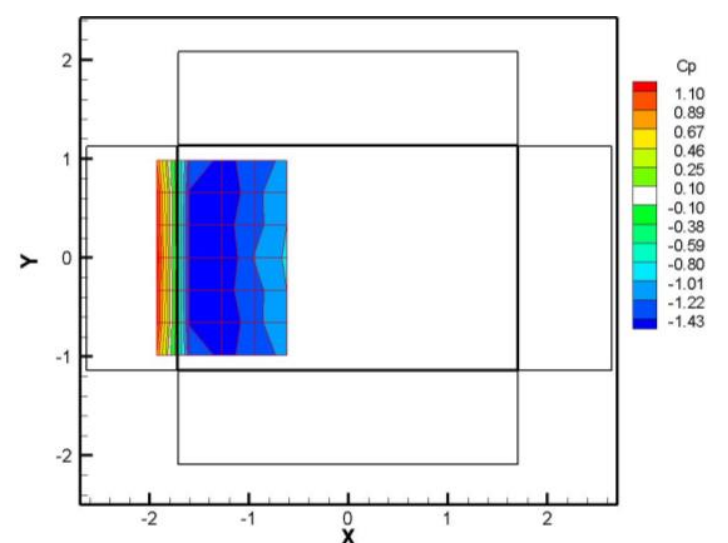

(b)

Fig. 15. Front wall with six rectangular blades streamlined with the flow and distributed at the front wall top edge with no rectangles at the corners

Trying to reduce the mass flow rate reaching the building roof, two configurations were tested. Figure 16 and Figure 17 respectively show the model with an overhang, and with a wing shaped device placed upstream at the building. It can be seen from Figure 16(b) and Figure 17(b) that the building with overhang resulted in low vacuum pressure than that with wing device. However, the aerodynamic forces on the building with overhang impose more structural load on the building and on the rigidity of the overhang and the roof than the forces associated with the wing device. On the other hand, the wing-shaped device redirects the incoming flow downward to align with the ongoing horseshoe vortex. Although this case did not reduce the peak vacuum much, it resulted in depletion of peak vacuum to a narrow span-wise strip behind the leading edge on the roof. It worth noting that the $\mathrm{Cp}$ measured on the façade at about $0.8 \mathrm{H}$ is about 0.5 while it appears to be around 0.9 or above for most of other the cases under consideration. This implies significant reduction of the aerodynamic forces and moments exerted on the building via its façade.

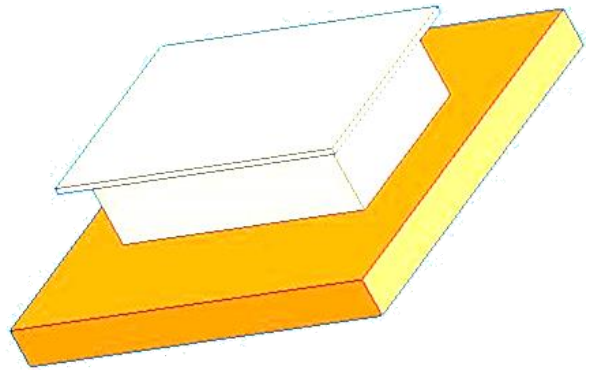

(a)

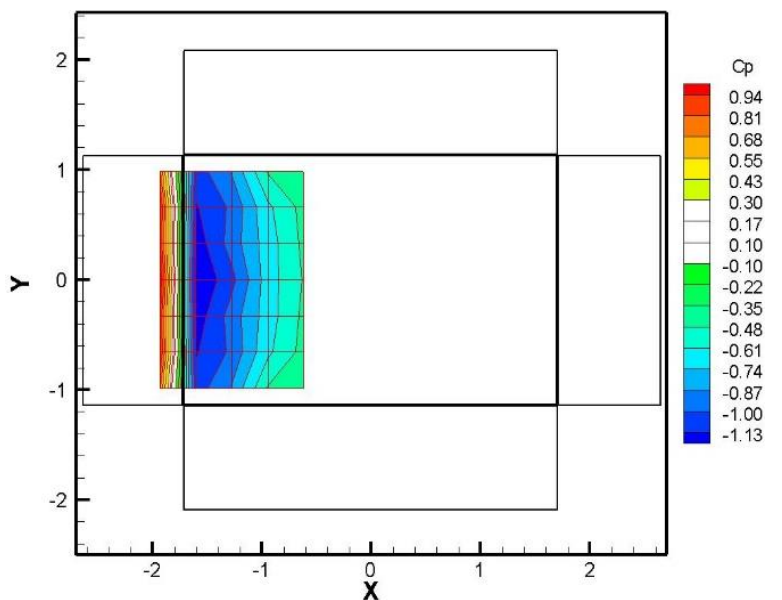

(b)

Fig. 16. Building with overhang 


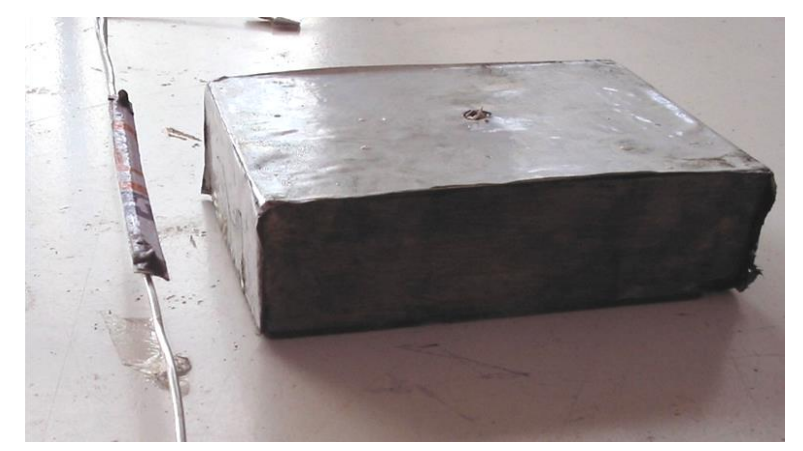

(a)

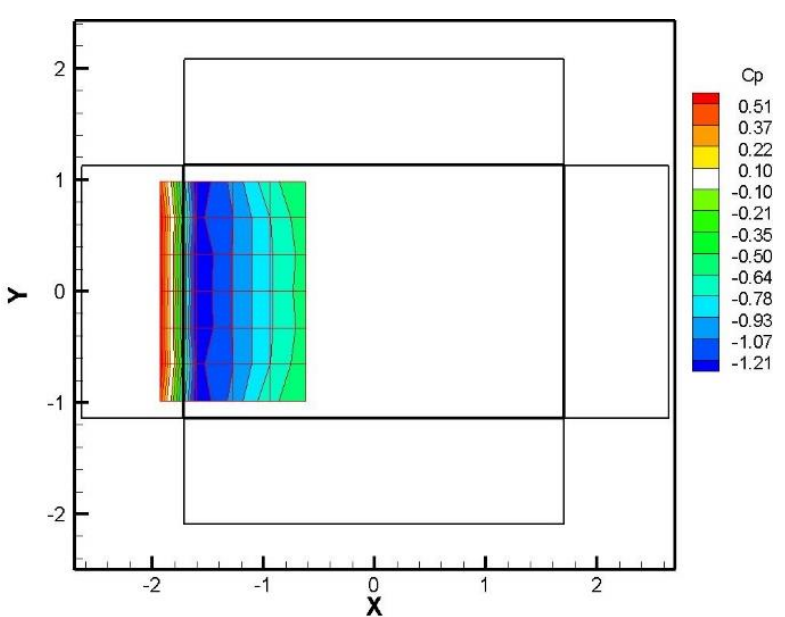

(b)

Fig. 17. Model with $7.5 \mathrm{~mm}$ thin airfoil placed upstream

\section{Conclusions}

A number of architectural modifications were proposed to improve the aerodynamic performance of low-rise buildings. Reduction of peak vacuum and redistribution of the pressure field over the building roof and on the building façade were reported. The cases of streamwise blades installed on the roof, Figure 12 and Figure 13, showed significant reduction in the peak vacuum on the roof. Additionally, the case of wing-shaped device, Figure 17, and the case of narrow curved strips over triangular blades, Figure 17, showed considerable reduction and improved distribution of the pressure field on the building roof as well as reduction of the positive pressure on the façade in the vicinity of the building height which indicate that the stagnation line assumed a lower level on the building façade. High vacuum occupying a relatively large area was observed on the roof when rectangular blades are installed to the upper part of the façade. This may be of interest to heat transfer applications. Although improvements to the pressure distributions and to the observed peak pressures are attained via different proposed configurations, the effects of such modifications on the statistics of the velocity field and the peak vacuum need to be investigated.

\section{Acknowledgments}

Thanks to the laboratory team at the Department of Mechanical Power Engineering, Zagazig University, for their good cooperation during the completion of the present research during the 2018 summer vacation.

\section{References}

[1] Ganesan, Timothy, and Mokhtar Awang. "Large Eddy Simulation (LES) of a Steady Turbulent Flow over a SurfaceMounted Cube." CFD Letters 4, no. 1 (2012): 1-9.

[2] Meile, Walter, Günter Brenn, Aaron Reppenhagen, Bernhard Lechner, and Anton Fuchs. "Experiments and numerical simulations on the aerodynamics of the Ahmed body." CFD letters 3, no. 1 (2011): 32-39.

[3] Aboshosha, Haitham, Girma Bitsuamlak, and Ashraf El Damatty. "LES of ABL flow in the built-environment using roughness modeled by fractal surfaces." Sustainable Cities and Society 19 (2015): 46-60. https://doi.org/10.1016/i.scs.2015.07.003

[4] Blocken, Bert, Ted Stathopoulos, Jan Carmeliet, and Jan LM Hensen. "Application of computational fluid dynamics in building performance simulation for the outdoor environment: an overview." Journal of Building Performance Simulation 4, no. 2 (2011): 157-184. https://doi.org/10.1080/19401493.2010.513740

[5] Yu, Dahai, Kyle Butler, Ahsan Kareem, James Glimm, and Jiangang Sun. "Simulation of the influence of aspect ratio on the aerodynamics of rectangular prisms." Journal of Engineering Mechanics 139, no. 4 (2013): $429-438$. https://doi.org/10.1061/(ASCE)EM.1943-7889.0000494 
[6] Kwon, Dae Kun, and Ahsan Kareem. "Towards codification of thunderstorm/downburst using gust front factor: Model-based and data-driven perspectives." Engineering Structures 199 (2019): 109608. https://doi.org/10.1016/j.engstruct.2019.109608

[7] El-Okda, Y. M., S. A. Ragab, and M. R. Hajj. "Large-eddy simulation of flow over a surface-mounted prism using a high-order finite-difference scheme." Journal of Wind Engineering and Industrial Aerodynamics 96, no. 6-7 (2008): 900-912. https://doi.org/10.1016/i.jweia.2007.06.017

[8] Lim, Hee Chang, T. G. Thomas, and Ian P. Castro. "Flow around a cube in a turbulent boundary layer: LES and experiment." Journal of Wind Engineering and Industrial Aerodynamics 97, no. 2 (2009): 96-109. https://doi.org/10.1016/i.jweia.2009.01.001

[9] Liu, Benli, Jianjun Qu, Weimin Zhang, Lihai Tan, and Yanhong Gao. "Numerical evaluation of the scale problem on the wind flow of a windbreak." Scientific Reports 4, no. 1 (2014): 1-5. https://doi.org/10.1038/srep06619

[10] Baskaran, A., S. Molleti, and D. Roodvoets. "Understanding low-sloped roofs under hurricane Charley from field to practice." In Roofing Research and Standards Development: 6th Volume. ASTM International, 2007. https://doi.org/10.1520/JAl101055

[11] Ilgın, H. Emre, and M. Halis Günel. "The role of aerodynamic modifications in the form of tall buildings against wind excitation." Metu Jfa 24, no. 2 (2007): 17-25.

[12] Kumar, Nallapaneni Manoj. "Performance of single-sloped pitched roof cadmium telluride (CdTe) buildingintegrated photovoltaic system in tropical weather conditions." Beni-Suef University Journal of Basic and Applied Sciences 8, no. 1 (2019): 1-9. https://doi.org/10.1186/s43088-019-0003-2

[13] John, Alok David, Gaurav Singla, Sumbul Shukla, and Rohit Dua. "Interference effect on wind loads on gable roof building." Procedia Engineering 14 (2011): 1776-1783. https://doi.org/10.1016/i.proeng.2011.07.223

[14] Soleimani, Zohreh, John Kaiser Calautit, and Ben Richard Hughes. "Computational analysis of natural ventilation flows in geodesic dome building in hot climates." Computation 4, no. 3 (2016): 31. https://doi.org/10.3390/computation4030031

[15] Zhao, Dong-Xue, and Bao-Jie He. "Effects of architectural shapes on surface wind pressure distribution: case studies of oval-shaped tall buildings." Journal of Building Engineering $12 \quad$ (2017): 219-228. https://doi.org/10.1016/i.jobe.2017.06.009

[16] Shamansurov, Javlon, and Kongratbay Sharipov. "Aerodynamic Analysis and Optimization of Passenger Bus ISUZU SAZ HC 40 and It's Impact on Fuil Economy." Acta of Turin Polytechnic University in Tashkent 9, no. 4 (2019): 148151.

[17] Meroney, Robert N., and David E. Neff. "Wind effects on roof-mounted solar photovoltaic arrays: CFD and windtunnel evaluation." In The Fifth International Symposium on Computational Wind Engineering (CWE 2010). 2010.

[18] Jain, Nishesh, Esfand Burman, Craig Robertson, Samuel Stamp, Clive Shrubsole, Francesco Aletta, Edward Barrett et al. "Building performance evaluation: Balancing energy and indoor environmental quality in a UK school building." Building Services Engineering Research and Technology 41, no. 3 (2020): 343-360. https://doi.org/10.1177/0143624419897397

[19] El-Okda, Yasser Mohamed. Experimental and numerical investigations of the effects of incident turbulence on the flow over a surface-mounted prism. Virginia Polytechnic Institute and State University, 2005.

[20] Josserand, Ch, and M. Rossi. "The merging of two co-rotating vortices: a numerical study." European Journal of Mechanics-B/Fluids 26, no. 6 (2007): 779-794. https://doi.org/10.1016/i.euromechflu.2007.02.005

[21] Iaccarino, G., A. Ooi, P. A. Durbin, and M. Behnia. "Reynolds averaged simulation of unsteady separated flow." International Journal of Heat and Fluid Flow 24, no. 2 (2003): 147-156. https://doi.org/10.1016/S0142727X(02)00210-2 